\title{
TRAUMATISMOS CAUSADOS POR EL TRÁNSITO EN PAÍSES EN DESARROLLO: AGENDA DE INVESTIGACIÓN Y DE ACCIÓN
}

\author{
Cheng-Min Huang ${ }^{1, a}$, Jeffrey C. Lunnen ${ }^{1, b}$, J. Jaime Mirandaª, ${ }^{2,3,}$ Adnan A. Hyder ${ }^{1, d}$
}

\begin{abstract}
RESUMEN
Los traumatismos causados por el tránsito son la principal causa de muerte en personas de 10-24 años a nivel mundial y representan alrededor del $15 \%$ de todas las muertes en varones. La carga de enfermedad de los traumatismos causados por el tránsito está distribuida de manera desigual entre los países pues la tasa de mortalidad más alta es ochenta veces superior a las más baja. Existe una clara desigualdad en el riesgo de ocurrencia de traumatismos causados por el tránsito, siendo notoriamente mayor en los países en desarrollo. Esta desigualdad se constituye como un reto mundial importante y se debe, aunque no sean los únicos factores, a muchas razones, incluyendo la rápida motorización y la pobre infraestructura. Este artículo hace énfasis en varios aspectos fundamentales cuya finalidad es informar a los programas diseñados para prevenir los traumatismos causados por el tránsito en los países en desarrollo, donde esta situación está más extendida. En primer lugar, la seguridad vial es un tema de desarrollo; en segundo lugar, los traumatismos causados por el tránsito constituyen un problema importante para la salud; en tercer lugar, los traumatismos causados por el tránsito pueden ser prevenidos mediante la implementación de medidas científicas adecuadas; en cuarto lugar, es necesaria la atención de emergencias hospitalarias y prehospitalarias; y, finalmente, la investigación en los traumatismos causados por el tránsito está relegada en los países de ingresos bajos y medios. Se discute además la repercusión de estos avances para el Perú.
\end{abstract}

Palabras clave. Accidentes de tránsito; Países en desarrollo; Salud pública (fuente: DeCS BIREME).

\section{ROAD TRAFFIC INJURIES IN DEVELOPING COUNTRIES: RESEARCH AND ACTION AGENDA}

\begin{abstract}
Road traffic injury (RTI) is the leading cause of death in persons aged $10-24$ worldwide and accounts for about $15 \%$ of all male deaths. The burden of RTI is unevenly distributed amongst countries with over eighty-fold differences between the highest and lowest death rates. Thus the unequal risk of RTI occurring in the developing world, due to many reasons, including but not limited to rapid motorization and poor infrastructure, is a major global challenge. This editorial highlights a number of key issues that must inform programs designed to prevent RTI in the developing world, where the epidemic is all the more insidious. Firstly, road safety is a development issue; secondly, road traffic injury is a major health issue; thirdly, road traffic injuries can be prevented by the implementation of scientific measures; fourthly, pre-hospital and hospital emergency care is needed; and fifthly, research on RTI is neglected in low-income and middle-income countries. The repercussion of such progress to Peru is also discussed.
\end{abstract}

Key words. Accidents, traffic; Developing countries; Public health (source: MeSH NLM).

\section{INTRODUCCIÓN}

Los traumatismos causados por el tránsito son la principal causa de muerte en personas de 10-24 años a nivel mundial y representan alrededor del $15 \%$ de todas las muertes en varones ${ }^{(1,2)}$. La carga de enfermedad de los traumatismos causados por el tránsito está distribuida de manera desigual entre los países pues la tasa de mortalidad más alta es ochenta veces superior a la mas baja ${ }^{(3)}$. Alrededor del $90 \%$ de traumatismos causados por el tránsito ocurren en países de ingresos bajos y medios ${ }^{(4)}$. Estos traumatismos causan muerte y discapacidad en varios grupos, incluyendo personas asalariadas, dejando a millones en la pobreza y a sus respectivas familias sin un soporte económico vital ${ }^{(5)}$. Existe una clara desigualdad en el riesgo de ocurrencia de traumatismos causados por el tránsito, siendo notoriamente mayor en los países en desarrollo. Esta desigualdad se constituye en un reto mundial

\footnotetext{
International Injury Research Unit, Department of International Health, Johns Hopkins Bloomberg School of Public Health. Baltimore, MD, USA.

Programa de Investigación en Accidentes de Tránsito, Salud Sin Límites Perú. Lima, Perú.

Facultad de Medicina, Universidad Peruana Cayetano Heredia. Lima, Perú.

a Médico, Magíster en Ciencias de la Salud; ${ }^{\text {b } C a n d i d a t o ~ a ~ M a g i ́ s t e r ~ e n ~ E s t u d i o s ~ d e ~ l a ~ M u j e r ; ~}{ }^{\circ}$ Médico, Magíster y Doctor en Epidemiología; ${ }^{\text {a }}$ Médico, Magíster y Doctor en Salud Pública.
}

Recibido: 19-02-10 Aprobado: 05-06-10 
importante y se debe, aunque no sean los únicos factores, a muchas razones incluyendo la rápida motorización y la pobre infraestructura.

La presencia de este reto, de una mayor vulnerabilidad vinculada con los traumatismos causados por el tránsito en países en vías de desarrollo, no necesariamente quiere indicar que la comunidad internacional se ha mantenido ajena a este desafío. En el año 2004, con la publicación del Informe mundial sobre prevención de los traumatismos causados por el tránsito elaborado por la Organización Mundial de la Salud (OMS) y el Banco Mundial, y una sesión de la Asamblea General de la Organización de las Naciones Unidas (ONU) dedicada a abordar la seguridad vial en el mundo, demostró ser una pieza fundamental en reconocer a los traumatismos causados por el tránsito como una epidemia. Recientemente, en el año 2009, este reporte fue seguido por el Informe sobre la situación mundial de la seguridad vial, elaborado por la OMS con el apoyo de la Bloomberg Family Foundation. Este último informe galvaniza esfuerzos para prevenir los traumatismos causados por el tránsito en 178 países, brindando una necesaria "línea de base" a nivel mundial ${ }^{(4)}$.

La OMS predice que las muertes vinculadas con los traumatismos causados por el tránsito aumentarán, debido al desarrollo económico, de 1,3 millones de personas en el año 2004 a 2,4 millones de personas en el año $2030{ }^{(6)}$. Según el Informe sobre la situación mundial de la seguridad vial, aproximadamente $90 \%$ de las muertes por traumatismos causados por el tránsito ocurren en países de ingresos bajos y medios, que albergan únicamente el $48 \%$ de los vehículos del mundo (4). Las insuficiencias en el diseño y el mantenimiento de infraestructuras, en la aplicación de la ley, en la atención de los traumas, e inclusive en los sistemas de vigilancia, son notorias en estos países ${ }^{(4)}$. Cabe señalar que en estos contextos el presupuesto financiero nacional para estos servicios es a menudo escaso.

Este artículo hace énfasis en varios aspectos fundamentales que deberían informar a los programas diseñados para prevenir los traumatismos causados por el tránsito en el mundo en desarrollo, donde la epidemia está más extendida. En primer lugar, la seguridad vial es un tema de desarrollo; en segundo lugar, los traumatismos causados por el tránsito constituyen un problema importante para la salud; en tercer lugar, los traumatismos causados por el tránsito pueden ser prevenidos mediante la implementación de medidas científicas adecuadas; en cuarto lugar, es necesaria la atención de emergencias hospitalarias y prehospitalarias; y, finalmente, la investigación en los traumatismos causados por el tránsito está relegada en los países de ingresos bajos y medios. Se discute, además, la repercusión de estos avances para el Perú.

\section{LA SEGURIDAD VIAL ES UN TEMA DE DESARROLLO}

La OMS, basada en evidencia sólida, promueve al menos cinco medidas clave para la prevención de los traumatismos causados por el tránsito: el uso obligatorio del cinturón de seguridad, el uso de sistemas de retención (asientos especiales) para niños, el uso del casco en ciclistas y motociclistas, la implementación de leyes vinculadas con el consumo de bebidas alcohólicas durante la conducción de vehículos y, la reducción de los límites de velocidad máxima. Según la OMS, el riesgo de morir en un accidente se reduce en $61 \%$ si se usa correctamente el cinturón de seguridad y el uso obligatorio de sistemas de retención para niños puede reducir las muertes infantiles, hasta en $35 \%$. El uso de casco reduce traumatismos craneales mortales y graves hasta en $45 \%$. El cumplimiento, en todo el mundo, de leyes vinculadas con el consumo de bebidas alcohólicas durante la conducción de vehículos podría reducir las colisiones relacionadas con el alcohol en $20 \%$. Y, por cada kilómetro que se reduce la velocidad promedio hay una reducción del $2 \%$ en el número de colisiones ${ }^{(7)}$.

De aplicarse estas medidas se reduciría significativamente el riesgo de resultar lastimado en una colisión de tránsito. Sin embargo, muchas veces la situación económica de la población representa un reto. En muchos países se ha descrito que mientras menor sea nivel socioeconómico de un individuo, mayor es el riesgo de sufrir un traumatismo vinculado con una colisión vehicular, se identifica así a la pobreza como un factor de riesgo importante ${ }^{(8,9)}$. Consecuentemente, aquellas familias que sobreviven día a día tratando de satisfacer necesidades y prioridades básicas, no tendrán acceso a este tipo de intervenciones efectivas sin la ayuda nacional e internacional.

Por otra parte, en los países de ingresos bajos y medios, las pérdidas ocasionadas por la muerte y discapacidad vinculada con los traumatismos causados por el tránsito pueden significar alrededor del $1-2 \%$ de su producto bruto interno ${ }^{(10)}$. En términos absolutos, esto podría sumar alrededor de US\$ 65 mil millones al año, que es más de lo que muchos países reciben en ayuda para el desarrollo. Si bien los programas de asistencia del extranjero apoyan a África, América Latina y Asia para reforzar sus infraestructuras, existe poco interés por adecuada planificación de los programas de seguridad vial a pesar de que estos podrían afectar potenciales ingresos provenientes del turismo, entre otras cosas. Por ejemplo, entre los años 2004 a 2006 se registraron 2361 muertes de ciudadanos de EE.UU. en el extranjero vinculadas con traumas, $50 \%$ de estas muertes ocurrieron en países del continente americano $y$, de estas, casi el $40 \%$, fueron consecuencia de traumatismos causados por el tránsito ${ }^{(11)}$. Invertir en el control de los traumatismos en el ámbito nacional puede transformar a los países de ingresos bajos y medios en lugares más seguros no sólo para los ciudadanos locales sino también para los extranjeros. 


\section{LOS TRAUMATISMOS CAUSADOS POR EL TRÁNSITO SON UN PROBLEMA IMPORTANTE DE SALUD}

Cada año más de 1,2 millones de personas mueren y hasta 50 millones de personas resultan heridas o discapacitadas por traumatismos causados por el tránsito. La OMS predice que los traumatismos causados por el tránsito se convertirán en la quinta causa de muerte en el año 2030. El Perú no es ajeno a este problema y recientemente los traumatismos causados por el tránsito han sido identificados como una causa importante de carga de enfermedad total en este país (12-14). Los traumatismos causados por el tránsito son una amenaza para la salud mundial y para el desarrollo de las sociedades, por lo que el papel de los organismos de seguridad vial merece una atención especial.

En la mayoría de los países existe la necesidad de contar con un organismo nacional que tenga suficiente financiamiento, personal adecuadamente entrenado y autoridad legal para recolectar información para evaluar y aplicar políticas de seguridad vial. Para ser efectivos, las políticas en seguridad vial en los países en desarrollo deben tener en cuenta las necesidades de todos los usuarios de las carreteras, sobre todo si se toman decisiones sobre la infraestructura, la planificación del uso de los terrenos y los servicios de transporte.

\section{LOS TRAUMATISMOS CAUSADOS POR EL TRÁNSITO PUEDEN PREVENIRSE CON MEDIDAS BASADAS EN LA EVIDENCIA}

La prevención de los traumatismos en general se basa tradicionalmente en el fundamento teórico de la matriz de Haddon en el que el huésped, el vector y los factores ambientales interactúan causalmente antes, durante y después del evento ${ }^{(15)}$. Dado que las enfermedades infecciosas y crónicas se pueden prevenir mediante la aplicación del modelo huésped-vector, creemos que la introducción de nuevas tecnologías y políticas eficaces reducirán los traumatismos causados por el tránsito. El Informe sobre la situación mundial de la seguridad vial (4) sugiere que las siguientes intervenciones pudieran ser implementadas: reduciendo la exposición al riesgo a través de políticas adecuadas de transporte y de uso de los terrenos, configurando la red de vial para la prevención de traumatismos causados por el tránsito, mejorando la visibilidad de los usuarios de las vías, promoviendo diseños de vehículos para prevención de lesiones, estableciendo y asegurando el cumplimiento de las normas de seguridad vial y brindando atención luego de la colisión ${ }^{(10,16)}$.

Como punto de partida, intervenciones tales como la aplicación más estricta del uso obligatorio del cinturón de seguridad, el uso de sistemas de retención (asientos especiales) para niños, el uso del casco en ciclistas y motociclistas, la implementación de leyes vinculadas con el consumo de bebidas alcohólicas durante la conducción de vehículos, y la reducción de los límites de velocidad máxima, tal y como ha sido indicado por la OMS, debe ser visto como un paquete comprensivo de intervenciones. Este paquete puede ser utilizado para promover el empoderamiento y la movilización de diversos agentes que podrían tener la función de mejorar la seguridad vial. Un aspecto clave para que estos esfuerzos den resultados es la implementación de estas medidas.

Sin embargo, los distintos contextos locales pueden plantear retos adicionales importantes, de manera que dicho paquete de intervenciones puede no resultar suficiente. Por ejemplo, en el Perú, a pesar de la existencia de límites de velocidad y leyes nacionales sobre el consumo de alcohol y conducción de vehículos, el $78 \%$ de las muertes relacionadas con traumatismos causados por el tránsito recae en el grupo de los peatones, el porcentaje más alto en la región (4). La seguridad vial no debería ser un privilegio que solamente gozan los usuarios de los vehículos. Mejores políticas e intervenciones basadas en evidencia sólida son esenciales para reducir los traumatismos causados por el tránsito en la mayoría de los "usuarios de vías públicas vulnerables" - los peatones- y los usuarios de los vehículos por igual.

El reto planteado por contextos particulares no quiere decir que la evidencia disponible no es aplicable. Por el contrario, adicionalmente a la implementación sistemática, estos contextos acusan la necesidad de una generación de evidencia complementaria. Esta debería ser rigurosa, innovadora y adecuadamente sustentada, desde las fases de investigación hasta su implementación. Este proceso debe ser visto como una inversión cuya rentabilidad repercutirá en un conjunto de intervenciones locales adecuadas destinadas a mitigar la carga de los traumatismos causados por el tránsito.

\section{ES NECESARIA LA ATENCIÓN PREHOSPITALARIA Y DE EMERGENCIA}

La encuesta del Informe sobre la situación mundial de la seguridad vial de la OMS mostró que $76 \%$ de los 178 países reporta alguna forma de sistema de atención prehospitalaria (4); sin embargo, la calidad de dichos sistemas no fue evaluada. Las diferencias observadas entre distintas regiones con relación a un desenlace más infortunado en aquellos pacientes que sufren traumas, pueden explicarse en parte por la falta de cirujanos capacitados, falta de personal de cuidados intensivos, por la presencia instalaciones médicas mal equipadas y por los servicios de emergencia y trauma desorganizados o inexistentes ${ }^{(17-20)}$. La mejora en la atención de emergencia prehospitalaria no es una tarea fácil, sin embargo, varios estudios revelan que la atención de trauma prehospitalaria en países de ingresos medios, 
puede ser mejorada con intervenciones de bajo costo, tal como ocurrió en México, por ejemplo ${ }^{(21)}$. Es hora de que la comunidad mundial invierta en la atención de trauma y en la atención prehospitalaria como una estrategia clave para mejorar la seguridad vial.

\section{LA INVESTIGACIÓN EN TRAUMATISMOS CAUSADOS POR EL TRÁNSITO ESTÁ RELEGADA}

Desde el Día Mundial de la Salud en el año 2004, el número de trabajos científicos sobre los traumatismos causados por el tránsito aumentó en los países de ingresos bajos y medios, sobre todo en el Lejano Oriente, el Medio Oriente y Europa. Sin embargo, en América Latina se observa una tendencia opuesta: la proporción de artículos publicados sobre traumatismos causados por el tránsito disminuyó en $49 \%$ desde el año $2004{ }^{222}$. Los países en desarrollo no sólo deberían tratar de reducir el número de traumatismos causados por el tránsito, fomentando la conducción de investigación sólida. Además, deberían también publicar dichas investigaciones para contribuir, de esta manera, con la masa de conocimiento mundial. Para ello, tanto los actores internacionales como las instituciones locales públicas y privadas, deben garantizar el apoyo adecuado para la generación de evidencia local.

El desarrollo de medidas efectivas de prevención de los traumatismos causados por el tránsito depende de información confiable y detallada acerca de las incidencias y los desenlaces de mecanismos específicos de los distintos tipos de trauma. La escasez de datos de vigilancia en los países en desarrollo conlleva a una subestimación de la magnitud del problema. De hecho, en el Informe sobre la situación mundial de la seguridad vial de la OMS ${ }^{(4)}$ sólo $22 \%$ de los países tenía información sobre la magnitud del problema de los traumatismos causados por el tránsito, los costos que estas lesiones ocasionan para el sector salud o para la economía nacional, así como datos necesarios para vigilar y evaluar adecuadamente las intervenciones puestas en marcha. Los sistemas de vigilancia en los países de ingresos bajos y medios podrían mejorarse de manera sustancial con inversiones selectivas ${ }^{(20)}$. Una actualización de los sistemas preexistentes, tales como datos de la policía, reportes de atención e internamiento en hospitales y los registros de mortalidad, podrían ser también efectivos.

\section{PROGRESOS Y REPERCUSIÓN PARA EL PERÚ}

Es crucial que los esfuerzos para llevar a cabo investigaciones, incluyendo el fortalecimiento de los sistemas de vigilancia, se amplíen en los países en desarrollo, teniendo en cuenta las especificidades de los contextos regionales. Investigación indispensable lo conforman la evaluación de los conocimientos, las actitudes y los comportamientos por parte del público; el establecimiento de prioridades regionales; la evaluación de costo-efectividad de las distintas intervenciones y la evaluación de las prácticas de atención hospitalaria existentes. Los grupos que vienen desarrollando o iniciando investigación en el tema deben ser reforzados y fortalecidos, y los investigadores locales deben ser alentados a compartir sus conocimientos a nivel internacional a fin de poder combatir la epidemia de traumatismos causados por accidentes de tránsito alrededor del mundo.

Además, los responsables de las políticas de salud deben establecer vías de comunicación con otros actores relevantes. Dos interesantes iniciativas locales merecen ser destacadas en el Perú; en primer lugar, el Ministerio de Salud realizó importantes progresos mediante el establecimiento, en el año 2004, de la Estrategia Sanitaria Nacional de Accidentes de Tránsito (ESNAT) ${ }^{(23)}$, similar a las de tuberculosis y de VIH/SIDA. A pesar de las limitaciones en su financiamiento, la ESNAT ha establecido un canal de intercambio entre varios actores locales, incluyendo varios sectores, entre ellos, policía, transporte, organizaciones no gubernamentales, organizaciones técnicas internacionales, proveedores de salud públicos y privados, entre otros. Aunque loable, persisten aún desafíos importantes relacionados, sobre todo, con identificar las estrategias más apropiadas para vincular e integrar efectivamente a aquellos tomadores de decisión de otros sectores, particularmente de los Ministerios de Economía y de Transportes.

En segundo lugar, la reciente identificación de las prioridades nacionales para la investigación en salud también ha sido importante. Aunque este ejercicio ha sido repetido recientemente de una manera más participativa, en el año 2007 el Instituto Nacional de Salud del Perú esbozó las prioridades de investigación a nivel nacional ${ }^{(24)}$, y, sobre todo, asignó financiamiento para llevar a cabo investigaciones en aquellas áreas identificadas como relegadas. Los traumatismos causados por el tránsito fueron seleccionados como una de esas áreas y, en el año 2009, se estableció el Programa de Investigaciones en Accidentes de Tránsito con financiamiento por un año ${ }^{(25)}$. Este liderazgo en el tema, originado desde el sector salud, necesita ser protegido, apoyado, sostenido y mejorado para asegurar su presencia y constancia en el tiempo y, puesto que tendrá impacto a corto, mediano y largo plazo en el progreso del área de los traumatismos causados por el tránsito en el Perú.

En América Latina, la mayoría de los países tienen leyes, al menos formuladas, sobre límites de velocidad, conducir en estado de ebriedad, uso de cascos, uso de cinturón de seguridad y de asientos especiales para niños. Sin embargo, carecen del poder para asegurar el cumplimiento efectivo de dichas leyes (4). Asimismo, existe una gran variabilidad, de manera que las altas incidencias de traumatismos causados por el 
tránsito seguirán marcando a la región. Como un tema de investigación, los investigadores deben apuntar a mejorar su comprensión de la dinámica entre pobreza y desigualdad como determinantes importantes de salud. El entender esta dinámica local resultará en la formulación de las políticas más adecuadas orientadas a remediar los traumatismos causados por el tránsito ${ }^{(26)}$.

\section{AGRADECIMIENTOS}

Nuestro agradecimiento a Ricardo Vásquez Orellana por su apoyo editorial en la elaboración de este manuscrito.

\section{Fuente de Financiamiento}

JJM fue el investigador principal del Programa de Investigación en Accidentes de Tránsito (PIAT), financiado por el Instituto Nacional Salud de Perú y ejecutado por la ONG Salud Sin Límites Perú.

\section{Conflicto de Interés}

Los autores declaran no tener conflictos de interés en la publicación de este artículo.

\section{REFERENCIAS BIBLIOGRÁFICAS}

1. Patton GC, Coffey C, Sawyer SM, Viner RM, Haller DM, Bose K, et al. Global patterns of mortality in young people: a systematic analysis of population health data. Lancet. 2009; 374(9693): 881-92.

2. Murray CJ, Lopez AD. Mortality by cause for eight regions of the world: Global Burden of Disease Study. Lancet 1997; 349(9061): 1269-76.

3. Kobusingye OC. Violence and injuries: What Africa should do. African Health Monit. 2008; 8(1): 37-40.

4. World Health Organization. Global status report on road safety: time for action. Geneva: WHO; 2009.

5. World Health Organization. The injury chart book: A graphical overview of the global burden of injuries. Geneva: WHO; 2002.

6. World Health Organization. The global burden of disease: 2004 update. Geneva: WHO; 2008.

7. World Health Organization. 10 facts about road safety: fact file [Página en Internet]. Geneva: WHO; 2009. [Fecha de acceso: junio 2009] Disponible en: http://www.who.int/ features/factfiles/roadsafety/en/.

8. Hyder AA. Road safety is no accident: a call for global action. Bull World Health Organ. 2004; 82(4): 240.

9. Nantulya VM, Reich MR. The neglected epidemic: road traffic injuries in developing countries. BMJ. 2002; 324(7346): 1139-41.

10. Peden M, Scurfiled R, Sleet D, Mohan D, Hyder AA, Jarawan E. World report on road traffic injury prevention. Geneva: World Health Organization; 2004.

11. Tonellato DJ, Guse CE, Hargarten SW. Injury deaths of US citizens abroad: new data source, old travel problem. J Travel Med. 2009; 16(5): 304-10.
12. Velásquez A. La carga de enfermedad y lesiones en el Perú y las prioridades del plan esencial de aseguramiento esencial. Rev Peru Med Exp Salud Publica. 2009; 26(2): 222-31.

13. Velásquez A. Análisis del estudio de carga de enfermedad en el Perú, MINSA-2004 y propuesta metodológica para el ajuste con datos nacionales de morbilidad. Lima: Promoviendo alianzas y estrategias, Abt Associates Inc.; 2006.

14. Perú, Ministerio de Salud, Dirección General de Epidemiología. Estudio de carga de enfermedad en el Perú-2004. Lima: MINSA, DGE; 2006.

15. Haddon W. Advances in the epidemiology of injuries as a basis for public-policy. Public Health Reports. 1980; 95(5): 411-21.

16. World Health Organization. Road traffic injury prevention: training manual. Geneva: WHO; 2006.

17. Arreola-Risa C, Mock C, Vega Rivera F, Romero Hicks E, Guzman Solana F, Porras Ramirez G, et al. Evaluating trauma care capabilities in Mexico with the World Health Organization's Guidelines for Essential Trauma Care publication. Rev Panam Salud Publica. 2006; 19(2): 94-103.

18. Mock C, Kobusingye O, Anh le V, Afukaar F, ArreolaRisa C. Human resources for the control of road traffic injury. Bull World Health Organ. 2005; 83(4): 294-300.

19. Mock C, Nguyen S, Quansah R, Arreola-Risa C, Viradia $\mathbf{R}$, Joshipura M. Evaluation of trauma care capabilities in four countries using the WHO-IATSIC Guidelines for Essential Trauma Care. World J Surg. 2006; 30(6): 94656.

20. Mock C, Quansah R, Krishnan R, Arreola-Risa C, Rivara F. Strengthening the prevention and care of injuries worldwide. Lancet. 2004; 363(9427): 2172-79.

21. Arreola-Risa C, Mock CN, Lojero-Wheatly L, de la Cruz O, Garcia C, Canavati-Ayub F, et al. Low-cost improvements in prehospital trauma care in a Latin American city. J Trauma. 2000; 48(1): 119-24.

22. Borse NN, Hyder AA. Call for more research on injury from the developing world: results of a bibliometric analysis. Indian J Med Res. 2009; 129(3): 321-26.

23. Perú, Ministerio de Salud. Estrategia Nacional de Accidentes de Tránsito [Página en Internet]. Lima: MINSA; 2009 [Fecha de acceso: 1 de junio de 2010]. Disponible en: http://www.minsa.gob.pe/portada/est_san/accidentes.htm.

24. Instituto Nacional de Salud. Prioridades de investigación en salud en el Perú: análisis del proceso. Lima: INS; 2007.

25. Instituto Nacional de Salud. Programa de Investigacion en Accidentes de Tránsito. [Página en Internet]. Lima: INS; 2009 [Fecha de acceso: 1 de junio de 2010]. Disponible en: http://www.ins.gob.pe/insVirtual/ins/ investigacionEnSalud/InvestigacionesFinanciadasINS/ programasInvestigacionAccidentesTransito.asp.

26. Caceres CF, Mendoza W. Globalized research and "national science": the case of Peru. Am J Public Health. 2009; 99(10): 1792-98.

\footnotetext{
Correspondencia: Adnan A. Hyder, MD MPH PhD

Dirección: 615 North Wolfe Street, Suite E-8132, Baltimore, Maryland, 21205, USA

Teléfono: +1-410-955-3928, Fax +1-410-614-1419

Correo electrónico: ahyder@jhsph.edu
} 